\title{
Si/SiGe HBT UWB Impulse Generators with Sleep-Mode Targeting the FCC Masks
}

\author{
Bernd Schleicher ${ }^{\ddagger}$, Jochen Dederer* and Hermann Schumacher ${ }^{\ddagger}$ \\ ${ }^{\ddagger}$ Institute of Electron Devices and Circuits, Ulm University, Albert-Einstein-Allee 45, \\ 89081 Ulm, Germany, Phone: +49-731-5031593, Email: bernd.schleicher@uni-ulm.de \\ *now with: EADS Deutschland GmbH, Wörthstraße 85, 89077 Ulm, Germany
}

\begin{abstract}
In this paper two UWB impulse generator ICs are presented, targeting the FCC ultra-wideband spectral masks for indoor and outdoor communications. For the generation of the impulse an underdamped RLC resonance circuit is excited by a short current spike. An on-chip power down circuit, consisting of a Widlar bandgap reference and several current mirrors was included, allowing to completely switch off the ICs with a single control signal under full DC biasing. The impulses show a very short time-domain extension and neglibile excess ringing, allowing high repetition rates, exceeding the $400 \mathrm{MHz}$ shown in this paper. The two impulse generators exhibit peak amplitudes of 170 and $180 \mathrm{mV}$ and the full-width at half maximum of the time domain waveforms are below 200 and $260 \mathrm{ps,} \mathrm{respectively.}$ All circuit blocks were realized using $0.8 \mu \mathrm{m} \mathrm{Si/SiGe} \mathrm{NPN} \mathrm{HBTs}$ only.
\end{abstract}

\section{INTRODUCTION}

In contrast to conventional carrier-based communication and radar systems, impulse radio ultra-wideband (IR-UWB) concepts use very short time-domain impulses without a carrier. The impulses, short in time and therefore occupying a wide bandwidth, are intended to fit into spectral masks, for example mandated by the Federal Communications Commission (FCC) in the U.S. [1] or the European Electronic Communications Committee (ECC) [2]. The impulses are therefore consisting of a section $\mathrm{T}_{\text {on }}$, where energy is present and a section $\mathrm{T}_{\text {off }}$, where no energy is present. Depending on the repetition rate of the impulses, which can be in the range of several $\mathrm{kHz}$ up to several hundreds of $\mathrm{MHz}$, and the impulse's width itself, very low duty cycles $\mathrm{T}_{\text {on }} / \mathrm{T}_{\text {off }}$ can be achieved. This inherent property of IR-UWB operation offers the potential to switch

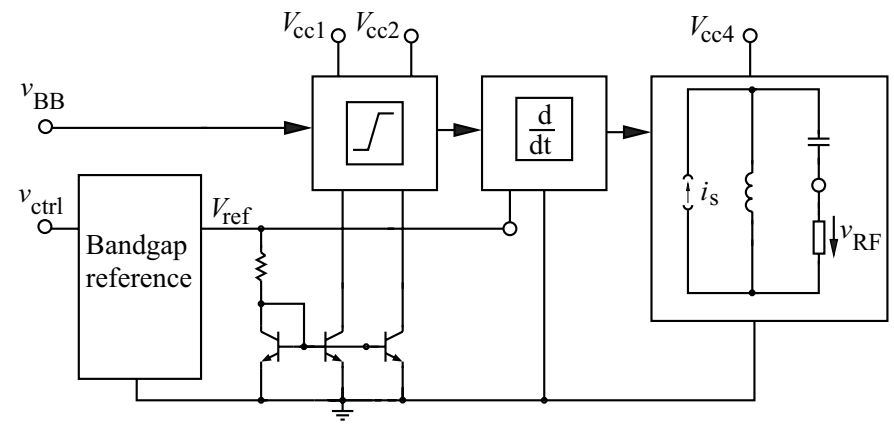

Fig. 1. Block diagram of the impulse generator circuit and the biasing concept for sleep-mode operation. off many circuit parts during $\mathrm{T}_{\text {off }}$, significantly reducing the average power absorbed from the DC supplies. Thus, IRUWB becomes a very interesting candidate in applications with low data rates or with burst-like transmissions with long dormant states in between, where a low power consumption is mandatory. An interesting example are communication components for medical implants, where data rate requirements are usually quite low and batteries have to operate for several years without the possibility of replacement, or any other sensor systems, where an "install and forget" approach is mandated.

One key element of every impulse generator is the formation of the radiatable UWB impulse itself. From [3], impulses using higher-order derivatives of a Gaussian impulse are suggested as good candidates for the time-domain impulse shape. Especially the fifth-order derivative with a $\sigma=51 \mathrm{ps}$ and the seventh-derivative with a $\sigma=65 \mathrm{ps}$ make efficient use of the allocated FCC indoor and outdoor masks, while having an adequate small time-domain extension. The implementation loss for fifth and seventh derivative Gaussian impulses compared to a hypothetical impulse completely filling the spectral mask from $3.1-10.6 \mathrm{GHz}$ is $2.9 \mathrm{~dB}$ and $3.9 \mathrm{~dB}$, respectively.

Promising circuit concepts in terms of low power consumption have been shown e.g. in [4], [5], where an oscillation is turned on and off by modulating the current control signal of a cross-coupled oscillator. In [6] the same principle to produce the impulse is used with a relaxation oscillator. Another principle to generate UWB impulses uses the excitation of a short spike-shaped relaxation transient in an underdamped resonant circuit (cf. [7]). This work uses the latter concept.

In all of these concepts, the part of the circuit controlling the impulse generation is the critical point. In [4]-[6] the control signal for impulse generation is formed using lowpower digital CMOS circuitry to produce a latch spike. This requires the presence of power efficient CMOS transistors in the semiconductor technology used, necessitating advanced RF CMOS or BiCMOS processes. The drawback when selecting one of these processes, generally, is their inherent high upfront cost, which makes them suitable only when market volumes are sufficiently large. The high up-front cost is due to the need for very fine lateral feature sizes, and, in case of BiCMOS, the large number of mask levels. At least in initial phases of system introduction with low market volumes, all NPN Si/SiGe HBT processes are advantageous due to their low 


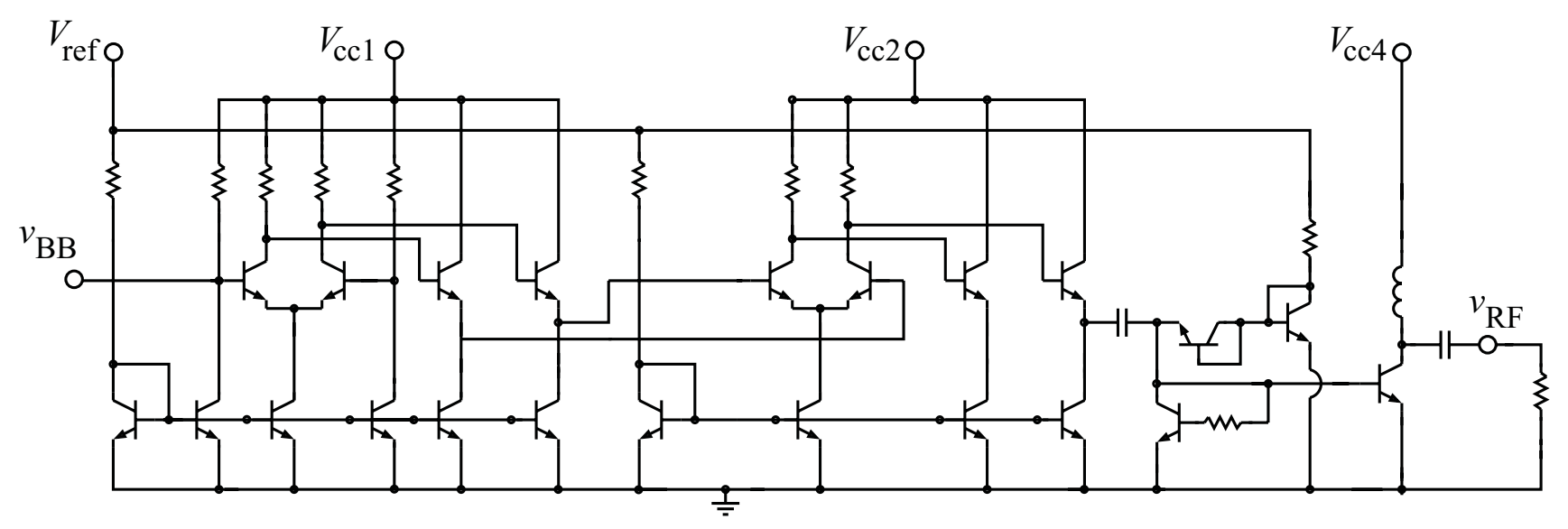

Fig. 2. Simplified circuit schematic of the impulse generator ICs. The bandgap reference has been omitted for clarity.

cost. However, a pure bipolar approach requires a different concept for spike generation. The aim of this paper is to show the operation of a monolithic impulse generator IC with a single on-chip sleep-mode control, which ensures a welldefined power-down capability of the circuit under full DC biasing.

\section{Si/Sige HBT Technology}

The semiconductor technology used is a commercially available pure $\mathrm{Si} / \mathrm{SiGe}$ heterojunction bipolar transistor (HBT) technology with a relaxed $0.8 \mu \mathrm{m}$ minimum drawn feature size [8]. The minimum effective emitter size on wafer for vertical NPN HBT transistors is $0.5 \times 1.1 \mu^{2}$. Two different types of NPN transistors are available. Transistors without selectively implanted collectors (non-SIC), have a transit frequency and maximum frequency of oscillation of $\mathrm{f}_{\mathrm{T}}=50 \mathrm{GHz}$ and $\mathrm{f}_{\max }=90 \mathrm{GHz}$, respectively, and a collectoremitter breakdown voltage $\mathrm{BV}_{\mathrm{CEO}}=4.3 \mathrm{~V}$. The second transistor type includes a selectively implanted collector (SIC) showing a transit frequency $\mathrm{f}_{\mathrm{T}}=80 \mathrm{GHz}$, a maximum frequency of oscillation $f_{\max }=90 \mathrm{GHz}$ and a collector-emitter breakdown voltage $\mathrm{BV}_{\mathrm{CEO}}=2.4 \mathrm{~V}$. Both types of transistors are used in the circuits presented here. The technology incorporates four types of resistance, metal-insulator-metal (MIM) and nitride capacitances, as well as a metal system with three metallization layers. Two kinds of silicon substrates are available, a standard low-resistivity $20 \Omega \mathrm{cm}$ substrate and a high-resistivity $1000 \Omega \mathrm{cm}$. The circuits presented here were fabricated on the low-resistivity substrate.

\section{CIRCUIT CONCEPT}

\section{A. Circuit Principle for Impulse Generation}

The fundamental concept of this impulse generator was discussed in detail in [7], [9], but is repeated here briefly for clarity and to indicate the neccessary changes for the sleep-mode operation. As can be seen in Fig. 1 the impulse generator consists of three main functional blocks. The first block comprises two limiting amplifiers, which are necessary to steepen the rising edges of an incoming digital signal
$\left(V_{B B}\right)$ and to obtain a signal with constant amplitude. Two limiting amplifiers are used to ensure operation even with low frequency sinusoidal input signals.

The rectangular signal is fed into the second building block, where it is differentiated, leading to positive and negative spikes with an approximated Gaussian shape. The negative spike, originating in the falling edge of the rectangular signal, is suppressed in the clamping circuit which is also part of the differentiator block. Each positive spike triggers a resonant RLC circuit, and causes an underdamped relaxation transient, producing the desired impulse shape. The resistive component of this RLC circuit is directly the $50 \Omega$ load resistance, or the appropriate feedpoint impedance of a wideband UWB antenna. By varying the inductance and capacitance values, the impulse shape can be adjusted in terms of relaxation frequency and impulse duration to make best use of the allocated spectral masks. Fig. 2 shows a simplified circuit schematic.

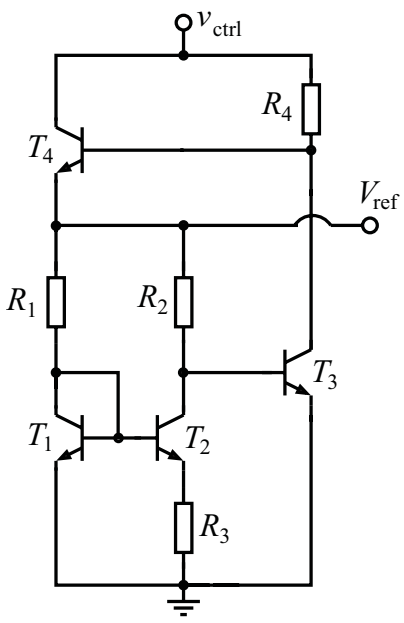

Fig. 3. Circuit schematic of the Widlar bandgap reference with $V_{c t r l}$ as input signal and $V_{\text {ref }}$ as resulting output signal. 


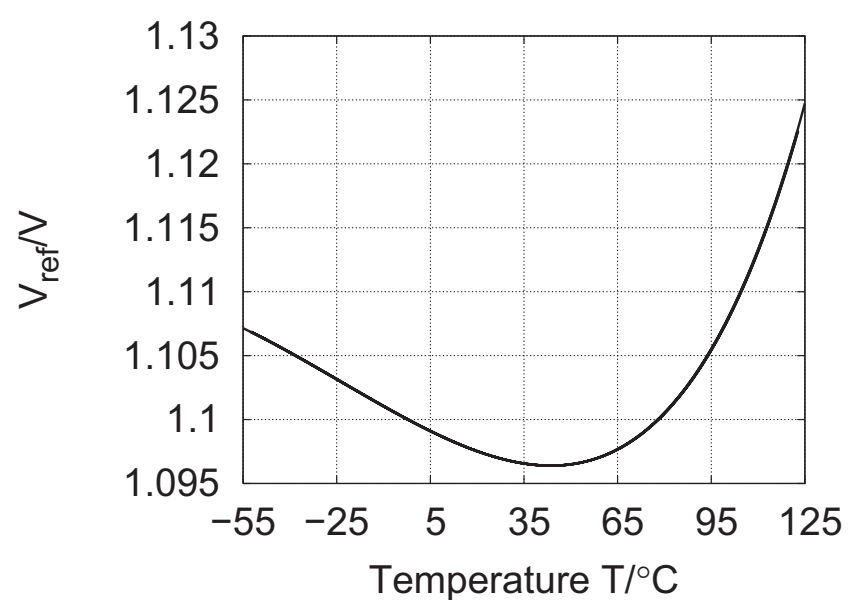

Fig. 4. Static simulation of the Widlar bandgap reference output voltage $V_{\text {ref }}$ as a function of temperature.

\section{B. Power-Down Operation and Bandgap Reference}

For the sleep-mode operation and in contrast to the previously published implementations of this concept, current mirrors were placed in all circuits blocks. They are used to switch off the circuit. As indicated in Fig. 1 a bandgap reference is used to provide a stable supply voltage reference $\left(V_{\text {ref }}\right) . V_{\text {ref }}$ as well supplies the differentiating circuit block. For the impulse generator stage, no additional power down capability is necessary, because the circuit only draws current when activated by the Gaussian shaped spike. When the bandgap reference control voltage $V_{c t r l}=0 \mathrm{~V}$, the circuit is completely switched off.

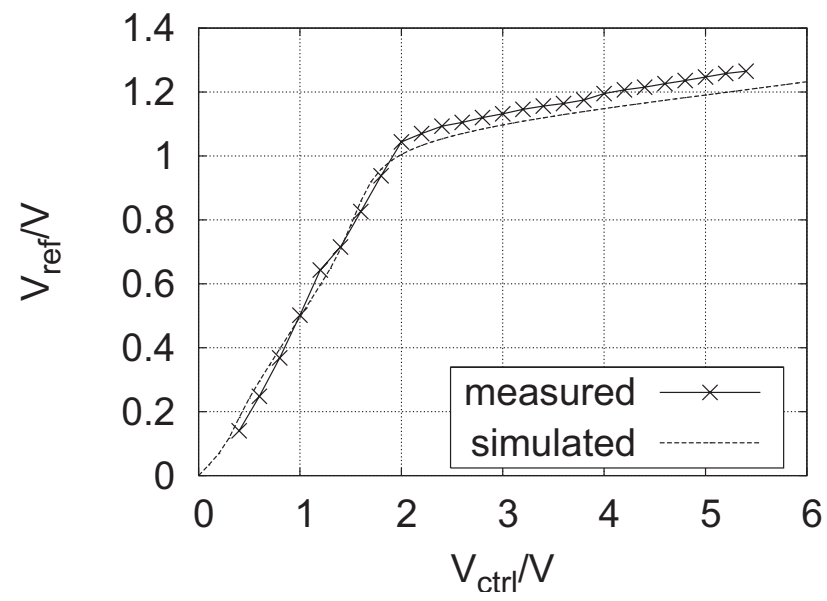

Fig. 5. Supply voltage dependence of the the Widlar bandgap reference voltage (simulation and measurement)

The circuit schematic of the Widlar bandgap reference used here is provided in Fig. 3. It shows an implementation similar to its simplest form as originally proposed in [10]. A bandgap reference establishes a stable voltage level $V_{\text {ref }}$ almost independent of the ambient temperature. As another benefit, $V_{r e f}$ is only weakly dependent on amplitude variations of the input signal, thereby removing stringent demands on accurate voltage levels in $V_{c t r l}$. The generated reference voltage $V_{\text {ref }}$ drives the current mirrors of the limiting amplifiers, which control the current flow.

\section{Simulation And Measurements}

\section{A. Bandgap Voltage}

Fig. 4 exhibits a simulation of the bandgap output voltage $V_{\text {ref }}$ versus temperature. In this simulation the temperature dependence of the transistors as well as the temperature dependence of the passive components is included. As can be seen, the output voltage is only a very weak function of temperature, changing only less than $10 \%$ over the full temperature range from -55 to $125^{\circ}$ Celsius.

Measurements of static performance characteristics of the bandgap reference circuit are carried out on a seperately placed test circuit on wafer. The input of the bandgap reference circuit $V_{c t r l}$ is biased with a standard voltage supply while the corresponding reference voltage $V_{\text {ref }}$ is measured with a highprecision voltmeter. Fig. 5 shows the comparison of measured and simulated reference voltages $V_{\text {ref }}$ for variations of $V_{c t r l}$ at room temperature. There is a maximum discrepancy of $5.2 \%$ between simulation and measurement at a $5.4 \mathrm{~V}$ control voltage, which can be very well attributed to the specified cross-wafer variations of component values. As desired, the bandgap reference voltage $V_{\text {ref }}$ is only weakly dependent on the applied input voltage $V_{c t r l}$.

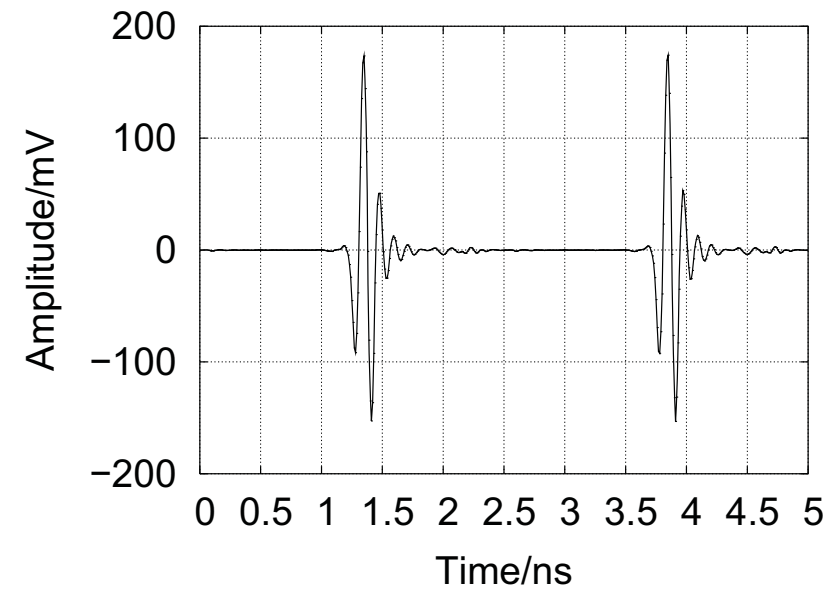

Fig. 6. $400 \mathrm{MHz}$ repetition rate impulses with an approximate $5^{\text {th }}$ order derivative Gaussian shape, measured on wafer.

\section{B. Measurements of the Impulse Shapes}

Measured time-domain waveforms of separate impulse generator MMICs with RLC values set to approximate a fifth and a seventh Gaussian derivative are depicted in Figs. 6 and 7. The different output waveforms are generated using an appropriate choice of the $\mathrm{LC}$ resonator elements. Here, the impulse generators are operated at elevated pulse repetition rates of $400 \mathrm{MHz}$. The peak amplitudes of the generated impulses are $170 \mathrm{mV}$ for the fifth Gaussian derivative and $180 \mathrm{mV}$ for the 


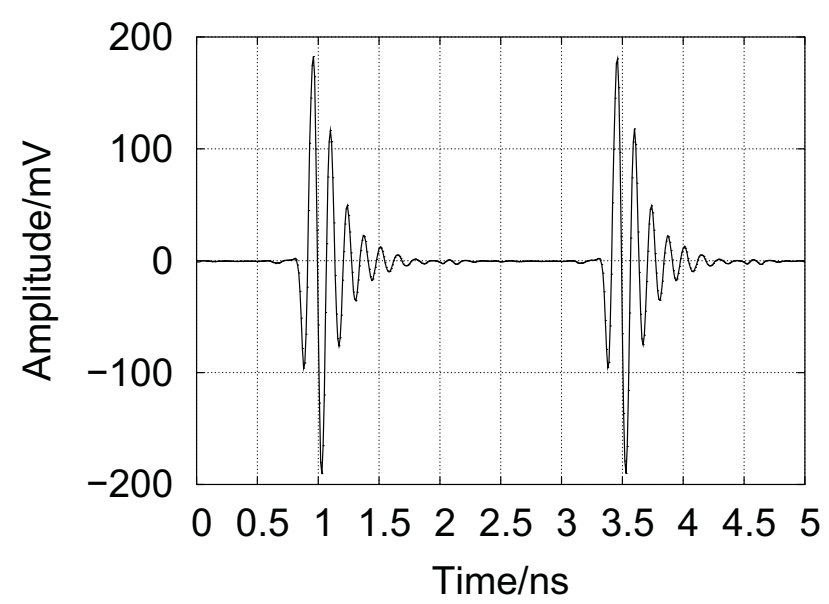

Fig. 7. Measurement of impulse trains as in Fig.6, but now for a circuit approximating the $7^{\text {th }}$ order derivative of a Gaussian pulse.

seventh Gaussian derivative impulse generator, whereas the full-width at half maximum (FWHM) time extension of the impulses is below $200 \mathrm{ps}$ for the fifth and below $260 \mathrm{ps}$ for the seventh Gaussian derivative impulse generator.

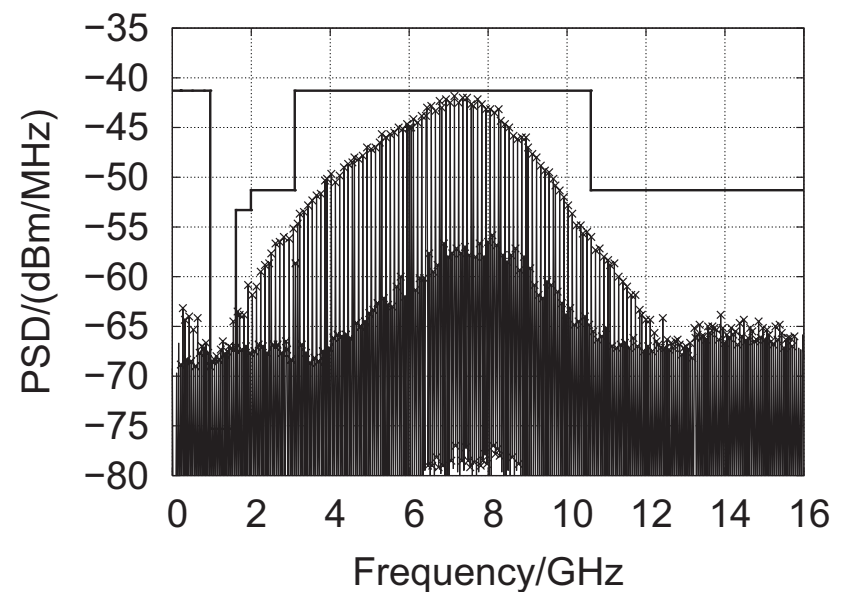

Fig. 8. Power spectral density (PSD) of $100 \mathrm{MHz}$ impulse trains generated by the fifth Gaussian derivative impulse generator, measured on wafer. The FCC mask for indoor communications is shown for comparison.

In the following, efficient use of the FCC emission limits for indoor and outdoor operation is exemplarily demonstrated with measured line spectra of impulse trains with $100 \mathrm{MHz}$ impulse repetition rates as shown in Figs. 8 and 9. The spectral performance can furthermore be improved by mounting the ICs to at the feed point of an antenna, using the antenna's bandpass characteristics to further improve spectral compliance with the masks as shown in [11].

Operated continuously at FCC emission limits with impulse repetition rates of $100 \mathrm{MHz}$, the fifth Gaussian derivative pulse generator exhibits an average DC power consumption of approximately $53 \mathrm{~mW}$, while the seventh Gaussian derivative pulse generator consumes an average of approximately $60 \mathrm{~mW}$.

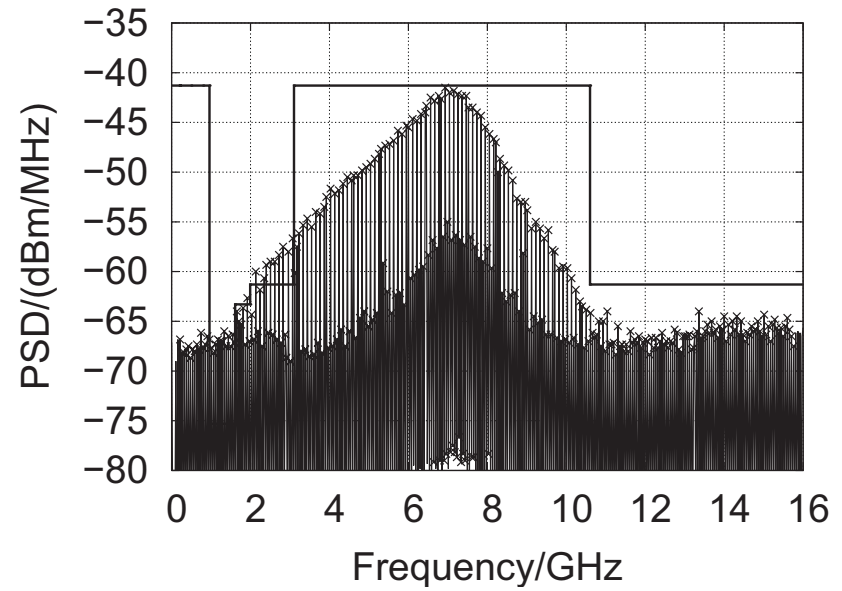

Fig. 9. PSD of $100 \mathrm{MHz}$ impulse trains generated by the seventh Gaussian derivative impulse generator, measured on wafer. The FCC mask for outdoor communications is shown for comparison.

However, as described above, the power consumption can be drastically reduced in case of low duty cycle applications by switching the pulse generator off during the time when no pulse energy is present. Measured leakage currents during offstate (sleep-mode) are in the nA-range and can be ignored compared to expected leakage currents of battery supplies.

Micrographs of the impulse generator ICs can be seen in Fig. 10. The fifth Gaussian derivative impulse generator IC has a chip area of $0.56 \times 0.57 \mathrm{~mm}^{2}$, while the seventh Gaussian derivative impulse generator measures $0.6 \times 0.61 \mathrm{~mm}^{2}$, both including bond pads.
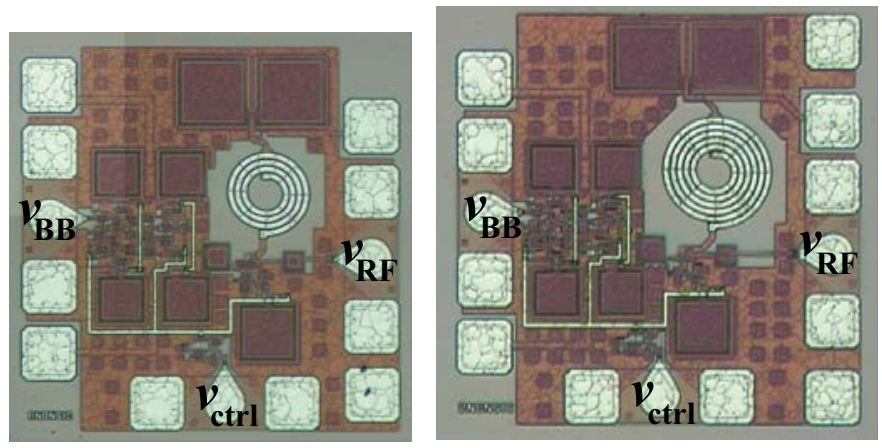

Fig. 10. Micrograph of the fifth (left) and seventh (right) Gaussian derivative impulse generator MMICs.

\section{CONCLUSION}

Two impulse generator ICs targeting the FCC spectral masks for UWB indoor and outdoor communications were demonstrated, using NPN transistors of an inexpensive $\mathrm{Si} / \mathrm{SiGe}$ HBT technology. Compared to previous designs, a powerdown capability was added which can dramatically reduce the average power consumption in low data rate or burst mode applications.

The measurements show good time domain performance with amplitudes of 170 and $180 \mathrm{mV}$ and a negligible excess 
ringing of the impulses at a full-width at half maximum (FWHM) of below 200 and $260 \mathrm{ps,} \mathrm{respectively.} \mathrm{The} \mathrm{ICs} \mathrm{make}$ efficient use of the allocated spectrum, despite their simple architecture. The impulse generators are suitable for repetition rates ranging from several $\mathrm{kHz}$ up to hundreds of $\mathrm{MHz}$ without an adjustment of the circuit.

\section{ACKNOWLEDGMENT}

The authors would like to thank ATMEL Automotive and Telefunken Semiconductors, both of Heilbronn, Germany, for their excellent support in the fabrication of the ICs. This project is funded by the German research foundation (DFG) by means of the priority programme UKoLoS.

\section{REFERENCES}

[1] Federal Communcations Commision, "Revision of Part 15 of the Commission's Rules Regarding Ultra-Wideband Transmission Systems," First report and order 02-48, FCC, Washington, D.C., USA, April 2002.

[2] Electronic Communications Committee (ECC), "COMMISSION DECISION of 21 February 2007 on allowing the use of the radio spectrum for equipment using ultra-wideband technology in a harmonised manner in the Community," document number C(2007) 522, European Conference of Postal and Telecommunications Administrations (CEPT), Feb. 2007.

[3] H. Sheng, P. Orlik, A. M. Haimovich, L. J. Cimini, and J. Zhang, "On the Spectral and Power Requirements for Ultra-Wideband Transmission," in IEEE International Conference on Communications (ICC), vol. 1, (Anchorage, AK, USA), pp. 738-742, May 2003.

[4] A. Cacciatori, L. Lorenzi, and L. Colalongo, "A Power Efficient HBT Pulse Generator for UWB Radars," in IEEE International Symposium on Circuits and Systems (ISCAS), (New Orleans, LA, USA), pp. 39163919, May 2007.

[5] A. T. Phan, J. Lee, V. Krizhanovskii, Q. Le, S.-K. Han, and S.-G Lee, "Energy-Efficient Low-Complexity CMOS Pulse Generator for Multiband UWB Impulse Radio," IEEE Transactions on Circuits and Systems, vol. 55, pp. 3552-3563, December 2008.

[6] J. R. Fernandes, H. B. Gonçalves, L. B. Oliveira, and M. M. Silva, "A Pulse Generator for UWB-IR Based on a Relaxation Oscillator," IEEE Transactions on Circuits and Systems, vol. 55, pp. 239-243, March 2008.

[7] J. Dederer, C. Schick, A. Trasser, and H. Schumacher, "A SiGe Impulse Generator for Single-Band Ultra-Wideband Applications," Semiconductor Science and Technology, vol. 22, pp. 200-203, December 2006.

[8] A. Schüppen, J. Berntgen, P. Maier, M. Tortschanoff, W. Kraus, and M. Averweg, "An $80 \mathrm{GHz}$ SiGe production technology," III-Vs Review, vol. 14, pp. 42-46, August 2001

[9] J. Dederer, B. Schleicher, F. de Andrade Tabarani Santos, A. Trasser, and H. Schumacher, "FCC compliant 3.1-10.6 GHz UWB Pulse Radar System using Correlation Detection," in IEEE MTT-S International Microwave Symposium (IMS), (Honolulu, Hawaii, USA), June 2007.

[10] R. J. Widlar, "New Developments in IC Voltage Regulators," IEEE Journal of Solid-State Circuits, vol. 6, no. 1, pp. 2-7, 1971.

[11] B. Schleicher, J. Dederer, M. Leib, I. Nasr, A. Trasser, W. Menzel, and H. Schumacher, "Highly Compact Impulse UWB Transmitter for HighResolution Movement Detection," in IEEE International Conference on Ultra-Wideband (ICUWB), vol. 1, (Hannover, Germany), pp. 89-92, September 2008. 